\title{
Representations of Groups Containing the Poincaré Group. I*
}

\author{
Robert Hermann \\ Applied Mathematics Division, Argonne National Laboratory \\ Received November 20, 1965
}

\begin{abstract}
It is proved that in certain representations of the Poincare group the mass operator must be identically zero.
\end{abstract}

\section{Introduction}

The aim of this series of papers is to study various questions related to the subject of the title, motivated in part by possible relevance to the application of group-theoretical ideas in elementary particle physics. Much of the work will be a continuation of that in [2].

Our immediate starting point is O'Raifeartaigh's theorem [5]. Let $P$ be the Poincaré (= inhomogeneous Lorentz) group, and let $G$ be a group containing $P$ as a subgroup. Consider an irreducible linear representation of $G$ in which the "mass" Casimir operator, $\square$, of $\mathbf{P}^{1}$ is Hermitian. O'RaIFEARTaIGH shows that no "mass splittings" are possible if $G$ is finite dimensional, i. e. if $\square$ has one discrete eigenvalue $m^{2}$, then $\square(\psi)=m^{2} \psi$ for all vector $\psi$.

This remarkable property of the Poincaré group has both negative and positive implications for physics. On the negative side, it indicates that many of the recent attempts to put particles of different masses into supermultiplets by a simple group-theoretic scheme are doomed to failure. Positively, it suggests a more elaborate scheme, whereby all particles of a certain type without interactions have the same mass, but the observed mass differences are the result of perturbations due to interactions.

The main purpose of this paper is to indicate further conditions on $G$ which imply that the eigenvalue $m^{2}$ must be zero. Roughly, this involves conditions on the normalizer of $T$ in $G$. ( $T$ is the group of trans-

* Work performed under the auspices of the United States Atomic Energy Commision.

1 The Lie algebra of a group will be denoted by the same letter in bold-faced type. For the notations used here, see [2].

Commun. math. Phys., Vol. 2 
lations in $P$. The normalizer of $\mathbf{T}$ in $\mathbf{G}$ is the set of all $Y \in \mathbf{G}$ such that $[Y, \mathbf{T}] \subset \mathbf{T}$.) It is not known whether these conditions are always satisfied; we conjecture that they are. At any rate, we will show that one general method for imbedding $P$ as a subgroup of larger groups does indeed lead to these conditions.

\section{The mass operator in representations of the Poincaré group}

Let $\mathbf{P}, \mathbf{T}$ and $\mathbf{G}$ be as described in the previous section. ( $\mathbf{G}$ is not necessarily finite dimensional, however.) We suppose that $G$ is a Lie algebra of operators on a Hilbert space $\mathfrak{G}$, with the elements of $\boldsymbol{T}$ represented by skew-Hermitian operators. Then, for $Y \in \mathfrak{G}, \psi \in \mathfrak{F}, Y(\psi)$ denotes the transform of $\psi$ by $Y$. Let $\left(X_{i}\right), 0 \leqq i \leqq 3$, be a basis for $\mathbf{T}$ in which the mass operator $\square$ takes the form:

$$
\square=\sum_{i} g_{i} X_{i}^{2}, \quad \text { with } g_{0}=-1, g_{i}=1 \text { for } i>0 .
$$

Let $Y$ be an operator of $\mathbf{G}$. Then,

$$
\begin{aligned}
\square Y(\psi) & =[\square, Y](\psi)+Y \square(\psi) \\
& =-\sum_{i} g_{i}\left(\left[Y, X_{i}\right] X_{i}+X_{i}\left[Y, X_{i}\right]\right)+Y \square(\psi) .
\end{aligned}
$$

This can be written in the form:

$$
\square Y(\psi)=\sum_{i} g_{i}\left(\operatorname{Ad} X_{i}\right)^{2}(Y)-2 \sum_{i} g_{i}\left[Y, X_{i}\right] X_{i}+Y \square(\psi) .
$$

Notice now that various simple assumptions about the commutation relations of the $X$ 's and $Y$ give information about the eigenvalues of $\square$.

For example, the simplest result of this type would be:

Theorem 2.1. If $\square$ is a scalar on $\mathfrak{G}$, i.e. $\square(\psi)=m^{2} \psi$ for all $\psi \in H$, and if:

$$
\left[Y, X_{i}\right]=\lambda X_{i} \text { for } 0 \leqq i \leqq 3, \text { with } \lambda \neq 0,
$$

then $m=0$.

The proof is obvious combining (2.1) and (2.3).

We shall show in Section 3 that (2.3) is satisfied for an element $Y$ of certain Lie algebras $\mathbf{G}$ into which $\mathbf{P}$ can be imbedded in a simple way.

Of course, O'Raifeartaigh's theorem gives a simple criterion that $\square$ be a scalar. We might remark that his condition can be extended slightly from the case where $\mathbf{G}$ is finite dimensional: namely, suppose $\mathbf{G}$ is the set of all operators $Y$ such that $\left(\operatorname{Ad} X_{i}\right)^{n}(Y)=0$ for $0 \leqq i \leqq 3, n$ sufficiently large. One proves easily that $\mathrm{G}$ is a Lie algebra of operators, and an obvious extension of O'Raifeartaigh's argument shows that if acting on $\mathfrak{G}$ has a discrete eigenvalue, than $G$ leaves invariant the eigenvectors corresponding to this eigenvalue. 
If one only assumes (2.3) is satisfied, there is still a considerable amount that can be said about $\square$. For

$$
[Y, \square]=\lambda \square
$$

and thus $Y$ and $\square$ together generate the Lie algebra of the two-parameter solvable group. All the representations of this group have been determined by GelfaNd and NaImark [4]. In particular, note that if does not have a discrete eigenvalue, it has a spectrum extending over the intervals $(-\infty, \infty),(0, \infty),(-\infty, 0)$. (I owe this remark to C. C. Moore.)

Now, we turn to deducing the most obvious consequences of (2.2). Suppose that $G$ is finite dimensional and semisimple. O'Raifeartaigh proves [5] that the transformation $\operatorname{Ad} X_{0}$ in $G$ is nilpotent. By the Morosov-Jacobson theorem in Lie algebra theory [3], there is then a $Y \in \mathbf{G}$ with

$$
\left[Y, X_{0}\right]=\lambda X_{0}, \text { and } \lambda \neq 0 \text {. }
$$

Suppose further that such a $Y$ can be found which is annihilated by $\sum_{i} g_{i}\left(\operatorname{Ad} X_{i}\right)^{2}$, i.e. by the mass Casimir operator of $\mathbf{P}$ in the adjoint representation of $\mathbf{P}$ in $\mathbf{G}$. Then, we can choose $^{2}$ a $\psi$ with $X_{i}(\psi)=0$ for $i=1,2,3$, i.e. a $\psi$ in the "rest frame." Then, if $\square(\psi)=m^{2} \psi$ for all $\psi \in \mathfrak{G}$, from $(2.2)$ we have

$$
m^{2} \lambda \psi=0 \text {, hence } m=0 .
$$

Finally, let us try to formulate a more general criterion that this sort of argument apply. Let $\mathbf{N}(\mathbf{T}, \mathbf{G})$ and $\mathbf{C}(\mathbf{T}, \mathbf{G})$, the normalizer and centralizer of $\mathbf{T}$ in $\mathbf{G}$, respectively, be the set of $Y \in \mathbf{G}$ such that $[Y, \mathbf{T}] \subset \mathbf{T}$ and $[Y, \mathbf{T}]=0$, respectively. Notice that:

$$
[\mathbf{C}(\mathbf{T}, \mathbf{G}), \mathbf{N}(\mathbf{T}, \mathbf{G})] \subset \mathbf{C}(\mathbf{T}, \mathbf{G}),
$$

i.e. $\mathbf{C}(\mathbf{T}, \mathbf{G})$ is an ideal in $\mathbf{N}(\mathbf{T}, \mathbf{G})$.

Let $\mathbf{L}$ be the subalgebra of $\mathbf{P}$ generated by the homogeneous Lorentz transformations. Now, we have:

Theorem 2.2. Suppose that:

a) $\square(\psi)=m^{2} \psi$ for all $\psi \in H$.

b) $\mathbf{N}(\mathbf{T}, \mathbf{G})$ is larger than $\mathbf{L}+\mathbf{C}(T, G)$.

Then, $m=0$.

Proof. Suppose that $Y \in \mathbf{N}(\mathbf{T}, \mathbf{G})$. Then

$$
\left[Y, X_{i}\right]=\sum_{j} a_{i j} X_{j}, \quad 0 \leqq i \leqq 3 .
$$

2 This is a point that admittedly should be made more precise, e.g. by the methods of "generalized eigenfunctions" of GELFAND and VaLENkIN [1]: The $X_{i}$ cannot be expected to have a discrete spectrum, so that $\psi$ is not in the Hilbert space. The point that must be justified is to show that it is permissible to act with $Y$ on $\psi$. 
Suppose that $\psi$ is an "eigenvector" of the $X_{i}$

$$
X_{i}(\psi)=b_{i} \psi, \quad 0 \leqq i \leqq 3 .
$$

(Note that at this point we are taking the same risk with rigor mentioned in the last footnote, namely assuming that eigenvectors of the $X_{i}$ can be added to $\mathfrak{S}$ in such a way that it makes sense to act on them with operators of $\mathbf{G}$.) Then, choosing this $\psi$ for 2.1 , we have:

$$
\sum_{i, j} g_{i} a_{i j} b_{i} b_{j}=0 \text {. }
$$

Now, if $Y \in \mathbf{L}$, the left hand side of 2.5 is identically zero in $b_{i}$, since Ad $Y$ preserves the metric tensor on $\mathbf{T}$. Let $Y \notin \mathbf{L}+\mathbf{C}(\mathbf{T}, \mathbf{G})$. The adjoint representation of $\mathbf{L}$ in $\mathbf{T}$ is just the usual $S O(3,1)$ representation of $L$, the Lorentz group. Hence, the left hand side of 2.5 is not identically zero in $b_{i}$, but only vanishes when:

$$
\sum_{i} g_{i} b_{i} b_{i}=m^{2} .
$$

This is clearly only possible if $m=0$.

\section{A method for generating groups containing the Poincaré group}

Let $\mathbf{P}=\mathbf{L} \oplus \mathbf{T}$ be the Lie algebra of the Poincaré group. Now, $\mathbf{P}$ is the contraction (see [2]) in the sense of Inonu-Segal-Wigner of various other Lie algebras; say that $\mathbf{F}$ is one of them. We propose a method for imbedding $\mathbf{P}$ in a Lie algebra $\mathbf{G}$ as follows: Find a one-parameter imbedding $\varepsilon \rightarrow \mathbf{F}_{\varepsilon}$ of $\mathbf{F}$ in $\mathbf{G}$ in such a way that, as $\varepsilon \rightarrow 0, \mathbf{F}_{\varepsilon}$ approaches (in the sense described in [2]) a subalgebra of $\mathbf{G}$ which is isomorphic to $\mathbf{P}$. We will not work at this level of generality in this paper: Instead, motivated by the detailed examples of this process worked out in [2], we will proceed in a more explicit manner.

Let us forget about $\mathbf{P}$ for the moment, and start off with a Lie algebra $\mathbf{G}$, a subalgebra $\mathbf{F}$ of $\mathbf{G}$, and an element $X \in \mathbf{G}$ such that $\operatorname{Ad} X$ acting in $G$ is completely reducible and has real eigenvalues. Let $\mathbf{C}(X)$ be the centralizer of $X$ in $G$, i.e. the subalgebra of elements $Z \in G$ with $[Z, X]=0$. Let $\mathbf{C}(X, \mathbf{F})$ be the centralizer of $X$ in $\mathbf{F}$, i.e.

$$
\mathbf{C}(X, \mathbf{F})=\mathbf{C}(X) \cap \mathbf{F} \text {. }
$$

Let $\mathbf{N}^{+}(X)$ be the subspace of $\mathbf{G}$ spanned by the eigenvectors of $\operatorname{Ad} X$ for positive eigenvalues. Then $\mathrm{N}^{+}(X)$ is a nilpotent subalgebra of $\mathbf{G}$. Further,

$$
\left[\mathbf{C}(X), \mathbf{N}^{+}(X)\right] \subset \mathbf{N}^{+}(X) .
$$

Notice that $\mathbf{C}(X, F)+\mathbf{N}^{+}(X)=\mathbf{P}(X)$ is a subalgebra of $\mathbf{G}$ that is a semidirect product of the subalgebra $\mathbf{C}(X, \mathbf{F})$ and the ideal $\mathbf{N}^{+}(X)$. We will now give a sufficient condition that $\mathbf{N}^{+}(X)$ be abelian, hence 
that $\mathbf{P}(X)$ have the same type of algebraic structure as the Poincare group.

Suppose that there is a subspace $\mathbf{M}$ of $\mathbf{G}$ such that:

$$
\mathbf{G}=\mathbf{F} \oplus \mathbf{M}, \quad X \in \mathbf{M},[\mathbf{C}(X, \mathbf{F}), \mathbf{M}] \subset \mathbf{M} .
$$

Then, the projection of $\mathbf{G}$ on $\mathbf{F}$ commutes with the action of $\operatorname{Ad} \mathbf{C}(X, \mathbf{F})$. Suppose further that the following conditions are satisfied

(I) $\operatorname{Ad} \mathbf{C}(X, \mathbf{F})$ acting in $\mathbf{F} / \mathbf{C}(X, \mathbf{F})$ is irreducible.

(II) If $Z \in \mathbf{F}$ and $[X, Z] \in \mathbf{F}$, then $Z \in \mathbf{C}(X, \mathbf{F})$.

Consider the projection of $\mathbf{N}^{+}(X)$ on $\mathbf{F}$, which we will call $\mathbf{F}(X)$. Then,

$$
[\mathbf{F}(X), \mathbf{C}(X, \mathbf{F})] \subset \mathbf{F}(X) .
$$

Project $\mathbf{F}(X)$ on $\mathbf{F} / \mathbf{C}(X, \mathbf{F})$. In view of $(\mathrm{I})$, there are two possibilities:

a) $\mathbf{F}(X) \subset \mathbf{C}(X, \mathbf{F})$, or

b) $\mathbf{F}=\mathbf{C}(X, \mathbf{F}) \oplus \mathbf{F}(X)$.

Let us consider a) first: Let $W \in \mathbf{N}^{+}(X)$, with

$$
[X, W]=\lambda W \text {. }
$$

Then, $W=Y+Z$, with $Y \in \mathbf{M}, Z \in \mathbf{F}$. Then,

$$
\lambda(Y+Z)=[X, Y]+[X, Z] .
$$

But, if condition a) is satisfied, $[X, Z]=0$. Hence, $\operatorname{Ad} X$ leaves invariant the subspace of $G$ spanned by $Y$ and $Z$. However, it does not act in a completely reducible way in this subspace, contradicting our assumption that $\operatorname{Ad} X$ is completely reducible. Then, a) is eliminated. Now, we can use the same argument to prove that:

Lemma 3.1. $\mathbf{F}(X) \cap \mathbf{C}(X, \mathbf{F})=(0)$, i.e., in view of $b)$,

$$
\mathbf{F}=\mathbf{C}(X, \mathbf{F}) \oplus \mathbf{F}(X) \text {. }
$$

Proof. Suppose otherwise, i.e. there is a relation of the form

Then,

$$
\begin{gathered}
W_{1}+\cdots+W_{\lambda}=Y+Z, Y \in \mathbf{M}, Z \in \mathbf{F}, \\
{[X, Z]=0 ; \quad\left[X, W_{i}\right]=\lambda_{i} W_{i} \text { for } i=1, \ldots, r .}
\end{gathered}
$$

$$
[X, Y]=\lambda_{l} W_{l}+\cdots+\lambda_{r} W_{r} .
$$

Consider the subspace of $\mathbf{G}$ spanned by $Y$ and $W_{l}, \ldots, W_{r}$. Ad $X$ leaves it invariant. Again, by complete reducibility of $\operatorname{Ad} X$, there should be a linear combination of $Y$ and $W_{l}, \ldots, W_{r}$ which is an eigenvector of $\operatorname{Ad} X$. This is clearly impossible.

Thus, the composite maps $\mathrm{N}^{+}(X) \rightarrow \mathbf{F}(X) \rightarrow \mathbf{F} / \mathbf{C}(X, \mathbf{F})$ is an isomorphism. In particular, in view of $(I)$ we have:

Corollary. Ad $\mathbf{C}(X, \mathbf{F})$ acts irreducibly in $\mathrm{N}^{+}(X)$.

As a consequence of this, we have: 
Theorem 3.1. $\mathrm{N}^{+}(X)$ is abelian, and $\operatorname{Ad} X$ acting on $\mathrm{N}^{+}(X)$ is just multiplication by a scalar.

Proof. $\left[\mathrm{N}^{+}(X), \mathbf{N}^{+}(X)\right]$ is a proper subspace of $\mathbf{N}^{+}(X)$ and is invariant under $\operatorname{Ad} \mathbf{C}(X, \mathbf{F})$, hence, in view of the Corollary to Lemma 3.1, must be zero. That $\operatorname{Ad} X$ is a scalar follows from Shur's Lemma.

Let us recapitulate this construction from a slightly different point of view. Start off with a Lie algebra $\mathbf{G}$, a subalgebra $\mathbf{F}$ such that there is a subspace $\mathbf{M}$ of $\mathbf{G}$ with:

$$
\mathbf{G}=\mathbf{F}+\mathbf{M}, \quad[\mathbf{F}, \mathbf{M}] \subset \mathbf{M} .
$$

Let $\mathbf{L}$ be a subalgebra of $\mathbf{F}$ such that $\operatorname{Ad} \mathbf{L}$ acting in $\mathbf{F} / \mathbf{L}$ is irreducible. Then, if $\mathbf{A d} \mathbf{L}$ acting in $\mathbf{M}$ has an invariant vector $X$ satisfying the relevant conditions, $\mathbf{L}+\mathbf{N}^{+}(X)$ is a subalgebra of $G$ consisting of a semidirect sum of an abelian ideal $\mathbf{N}^{+}(X)$ of "translations", together with a subalgebra $\mathbf{L}$ and a representation of $\mathbf{L}$ which is equivalent to the action of Ad $\mathbf{L}$ in $\mathbf{F} / \mathbf{L}$. Under further conditions described more carefully in [2], $\mathbf{L}+\mathbf{N}^{+}(X)$ will then be a contraction and a limit within $G$ of the subalgebra $\mathbf{F}$. Further, if $\mathbf{T}$ is put equal to $\mathbf{N}^{+}(X)$, we will be able to apply Theorem 2.1, since $X$ is in $\mathbf{N}(T, \mathbf{G})$, and $\mathbf{T}$ will have a basis on which $\operatorname{Ad} X$ is diagonal with real eigenvalues.

For example, let us apply this construction to the case

$$
L=S O(3,1), F=S O(4,1), \quad G=S O(4,2) .
$$

It is readily verified that all these conditions are satisfied, and we get $\mathbf{L}+\mathbf{N}^{+}(X)$ as $\mathbf{P}$, the Lie algebra of the Poincaré group. $G$ is of course just the conformal group of Minkowski space, so our imbedding of $\mathbf{P}$ in a larger group is just that which is most natural from a geometric point of view. Further, $S O(4,2)$ is locally isomorphic to $S U(2,2)$, the group of $4 \times 4$ unimodular complex matrices which preserve a Hermitian form with two plus and two minus signs. The Lie algebra of this group can be expressed in terms of the Dirac matrices, of course. This seems to be identical with the representation of $\mathbf{P}$ in terms of Dirac matrices used recently in the many attempts to render the $S U(6)$-theory of Gursey, Radacati and Sakita relativistically invariant.

We can make one final remark about these attempts. Suppose that we put together $P$ and another group $K$ by the "Wigner construction", namely choose representations $\varrho_{1}$ and $\varrho_{2}$ of $P$ and $K$ on vector spaces $V_{1}$ and $V_{2}$. Let $V=V_{1} \otimes V_{2}$, and construct a representation $\varrho$ of the direct sum Lie algebra $\mathbf{P} \oplus \mathbf{K}$ on $V$ by the rule:

$$
\varrho(X \oplus Z)\left(v_{1} \otimes v_{2}\right)=\varrho_{1}(X)\left(v_{1}\right) \otimes v_{2}+v_{2} \otimes \varrho_{2}\left(Z\left(v_{2}\right)\right) .
$$

Wigner's construction now consists in letting $\mathrm{G}$ be the smallest associative algebra of operators on $V$ containing $\varrho(P \oplus K)$. For example, if $P$ is imbedded in $S U(2,2)$ as explained above, and if $S U(2,2)$ is regarded 
in the usual way as a group of transformations on $C^{4}=V_{1}$, and if $K=S U(3)$, with $V_{2}=C^{3}$, then $V$ is $C^{12}$, and $G$ is $S U(6,6)$.

Now, one basic difficulty in interpreting this physically ${ }^{3}$ is that $\mathbf{T}$, the algebra of transformations in $\mathbf{P}$, is no longer maximal abelian in $\mathbf{G}$. The additional abelian operators would give new "quantum numbers" of particles which must be interpreted physically. If one "throws them away" by considering unitary representations of $\mathbf{G}$ on a Hilbert space $\mathfrak{S}$, then only considers subspaces of $\mathfrak{F}$ on which the additional operators are zero, one runs into the well-known "unitarity difficulties." Now, these additional abelian operators arise because $\varrho_{1}(X)$, for $X \in \mathbf{T}$, is nilpotent of second order, i.e. $\varrho_{1}(X) \varrho_{1}\left(X^{\prime}\right)=0$ for $X, X^{\prime} \in \mathbf{T}$. To see this, consider

$$
\varrho_{1}(X) \varrho_{\mathbf{2}}(Z), \text { for } \quad X \in \mathbf{T}, Z \in \mathbf{K} \text {. }
$$

It applied to $v_{1} \otimes v_{2}$ gives $\varrho_{1}(X)\left(v_{1}\right) \otimes \varrho_{2}(Z)\left(v_{2}\right)$.

$$
\text { Hence, } \begin{aligned}
& {\left[\varrho_{1}(X) \varrho_{2}(Z), \varrho_{1}\left(X^{\prime}\right) \varrho_{2}\left(Z^{\prime}\right)\right]\left(v_{1} \otimes v_{2}\right) } \\
&= \varrho_{1}(X) \varrho_{2}(Z) \varrho_{1}\left(X^{\prime}\right) \varrho_{2}\left(Z^{\prime}\right)\left(v_{1} \otimes v_{2}\right)- \\
&-\varrho_{1}\left(X^{\prime}\right) \varrho_{2}\left(Z^{\prime}\right) \varrho_{1}(X) \varrho_{2}(Z)\left(v_{1} \otimes v_{2}\right) \\
&= \varrho_{1}(X) \varrho_{1}\left(X^{\prime}\right)\left(v_{1}\right) \otimes \varrho_{2}(Z) \varrho_{2}\left(z^{\prime}\right)\left(v_{2}\right)- \\
&-\varrho_{1}\left(X^{\prime}\right) \varrho_{1}(X)\left(v_{1}\right) \otimes \varrho_{2}\left(Z^{\prime}\right) \varrho_{2}(Z)\left(v_{2}\right) \\
&= \varrho_{1}(X) \varrho_{1}\left(X^{\prime}\right)\left(v_{1}\right) \otimes \varrho_{2}\left(\left[Z, Z^{\prime}\right]\right)\left(v_{2}\right) .
\end{aligned}
$$

Thus, no matter what the structure of $K$, all these operators will be zero: This is the source of the "catastrophe," since it gives a huge number of additional abelian operators in $\mathbf{G}$ containing $\mathbf{T}$. However, if the representation $\varrho_{1}$ of $P$ were chosen so that the $\varrho_{1}(\mathbf{T})$ were nilpotent to a higher order than two, the number of such additional abelian operators would be cut down considerably. Such representations of $P$ have not yet been considered: Presumably they are in spaces of much higher dimension, and introduce other severe complications.

\section{Bibliography}

[1] Gelfand, I., and J. Valenkin: Generalized Functions, IV. New York: Academic Press 1964.

[2] Hermann, R.: Lie Groups for Physicists. New York: Benjamin 1965.

[3] JACOBSON, N.: Lie Algebras. New York: Interscience 1962.

[4] NaImark, M. A.: Normed Rings. Groningen: P. Nordhoff 1959.

[5] O'Ratfeartaigh, L.: Phys. Rev. Lettres 14, 575-577 (1965).

${ }^{3} \mathrm{I}$ am indebted to W. McGuINN for many conversations and insights concerning this and other points concerning the relation of the group theory and physics. 